\title{
Corpus Callosum Agenesis
}

National Cancer Institute

\section{Source}

National Cancer Institute. Corpus Callosum Agenesis. NCI Thesaurus. Code 698905.

A congenital abnormality characterized by the complete absence of the corpus callosum.

It may be an isolated abnormality or associated with other central nervous system abnormalities or syndromes. Clinical manifestations vary. In cases of isolated corpus callosum agenesis, symptoms may be absent or minimal. In cases that are associated with other central nervous system abnormalities or syndromes, symptoms include developmental delays, motor coordination difficulties, and vision impairment. 